\title{
Montmorillonite/chitosan nanoparticles as a novel controlled-release topical ophthalmic delivery system for the treatment of glaucoma
}

This article was published in the following Dove Press journal: International Journal of Nanomedicine

\author{
Juan Li,' Shuangyan Tian,' \\ Qi Tao, ${ }^{2}$ Yawen Zhao,' Ruyi \\ Gui,' Fan Yang,' Lingquan \\ Zang, ${ }^{3}$ Yanzhong Chen, ${ }^{4}$ \\ Qineng Ping, ${ }^{5}$ Dongzhi Hou' \\ 'Department of Pharmaceutics, \\ Guangdong Provincial Engineering \\ Center of Topical Precise Drug \\ Delivery System, Guangdong \\ Pharmaceutical University, Guangzhou, \\ People's Republic of China; ${ }^{2}$ CAS \\ Key Laboratory of Mineralogy and \\ Metallogeny \& Guangdong Provincial \\ Key Laboratory of Mineral Physics \\ and Materials, Guangzhou Institute \\ of Geochemistry, Chinese Academy \\ of Sciences, Guangzhou, People's \\ Republic of China; ${ }^{3}$ Department of \\ Pharmacology, College of Pharmacy, \\ Guangdong Pharmaceutical University, \\ Guangzhou, People's Republic of \\ China; ${ }^{4}$ Guangdong Provincial Key \\ Laboratory of Advanced Drug \\ Delivery Systems, Guangdong \\ Pharmaceutical University, Guangzhou, \\ People's Republic of China; ${ }^{5}$ College \\ of Pharmacy, China Pharmaceutical \\ University, Nanjing, People's Republic \\ of China
}

Background: To date, the rapid clearance from ocular surface has been a huge obstacle for using eye drops to treat glaucoma, since it has led to the short preocular residence time and low bioavailability.

Methods: The novel nanoparticles (NPs) were designed for topical ophthalmic controlled drug delivery system through intercalating the $\mathrm{BH}$ into the interlayer gallery of Na-montmorillonite $(\mathrm{Na}+\mathrm{Mt})$ and then further enchasing chitosan nanoparticles. The resulting nanoparticles had a positive charge $(+29 \pm 0.18 \mathrm{mV})$ with an average diameter of $460 \pm 0.6 \mathrm{~nm}$.

Results: In vitro study of drug release profiles suggested controlled release pattern. The irritation experiment analysis on both human immortalized cornea epithelial cell (iHCEC) and chorioallantoic membrane-trypan blue staining (CAM-TBS) showed good tolerance for ocular tissues. It was interestingly found that the nanoparticles could enter into iHCEC from the result of cellular uptake experiment measured by confocal layer scan microscopy (CLSM). Meanwhile, multilayered iHCEC was used to simulate the barrier of corneal epithelial cells for in vivo preocular retention capacity study, which suggested that BH-Mt/CS NPs could prolong the retention time in comparison with $\mathrm{BH}$ solution. The ocular pharmacokinetics studied by microdialysis sampling technique showed that $\mathrm{AUC}_{0-\mathrm{t}}$ and $\mathrm{MRT}_{0-\mathrm{t}}$ of $\mathrm{BH}-\mathrm{Mt} / \mathrm{CS}$ NPs were 1.99-fold and 1.75-fold higher than those of BH solution, indicating higher bioavailability. Moreover, the study of blood drug concentration, few researchers have reported, showed that low level drug could enter into blood, suggesting lower systematic side effect. Importantly, pharmacodynamics studies suggested that BH-Mt/CS NPs could make a significant decreased intraocular pressure on glaucomatous rabbits.

Conclusion: Inspired by these advance of montmorillonite/chitosan nanoparticles, we envision that the BH-Mt/CS NPs will be a potential carrier for BH, opening up the possible applications in glaucoma therapy.

Keywords: montmorillonite, glaucoma, chitosan, betaxolol hydrochloride, preocular retention

\section{Introduction}

Glaucoma is the second major cause of blindness, characterized by the increase of intraocular pressure (IOP). ${ }^{1}$ Continuous elevated IOP would cause eventual damage of the optic nerve and consequently loss of vision. ${ }^{2}$ Traditionally, for the topical ophthalmic formulations of glaucoma, there are two methods to reduce the IOP: 1) reducing the production of the aqueous humor and 2) increasing its outflow. ${ }^{3}$ For example, betaxolol hydrochloride $(\mathrm{BH})$, a selective beta-adrenergic blocking agent, is often used to increase outflow of aqueous humor to alleviate open-angle glaucoma. ${ }^{4}$ However, the $\mathrm{BH}$, as with other ophthalmic preparations, is often eliminated rapidly from precorneal area owing to the particularity of the eye, including the presence of
Department of Pharmaceutics,

Guangdong Provincial Engineering Center of Topical Precise Drug Delivery System, Guangdong Pharmaceutical University, 280 Daxuecheng Outer Ring East Road, Panyu, Guangzhou 510640 People's Republic of China

$\mathrm{Tel}+8618026312508$

Fax +86 2039352117

Email houdongzhi406@I63.com 
tissue barriers (cornea, lens, conjunctiva, and sclera) and the various physiological mechanisms (lacrimation, tear dilution, and tear turnover). ${ }^{5,6}$ Due to this particularity, a number of precorneal loss will result in low ocular bioavailability of drugs (only $<5 \%$ of administered drug could enter into the eye). ${ }^{7}$ Thus, traditional ophthalmic preparations cannot provide and maintain an adequate drug concentration in the corneal tissue and finally result in a poor ocular bioavailability. To solve such a conundrum, frequent dosing is required for glaucoma patients. Nevertheless, it would lead to poor patient compliance and systemic side effect, especially for patients with severe renal impairment. ${ }^{8}$ Hence, there is urgent need to find an ingenious scheme to improve bioavailability.

It is generally considered that ocular bioavailability would be tremendously improved if the corneal permeability time and precorneal residence of drug could be increased. ${ }^{9}$ However, the strategy to improve corneal permeability is mainly achieved by the addition of penetration enhancer agents, which may cause some toxicity to eye. Therefore, many research efforts have been focused on using materials to develop advanced systems with good adhesion properties, which could improve precorneal residence time and obtain a desired bioavailability. For instance, nanoparticles (NPs) have been used as an alternative carrier to traditional delivery systems in ophthalmology due to their excellent potential advantage, such as low irritation and enhanced adsorption. ${ }^{10,11}$ In order to further improve the contact with eye mucosa, the properties of NPs were formed by using cationic or mucoadhesive polymers (chitosan, sodium alginate, xanthan gums, etc.). ${ }^{12,13}$ For these, chitosan has been widely used as a pharmaceutical excipient for ocular application due to its superior characteristics, such as low irritation, biodegradability, biocompatibility, and long resident time in the corneal surface. ${ }^{14}$ Meanwhile, chitosan has an amino group that could readily undergo protonation in acidic to neutral conditions, resulting in a net positive charge. Also, the positively charged chitosan can bind to the negatively charged corneal mucin, which is beneficial to prolong the precorneal retention time of chitosan-bound drugs, leading to enhanced bioavailability. ${ }^{15}$ In early studies, Cho et al used chitosan as a promising cationic biomaterial for ophthalmic preparation, and the formulation showed significant therapeutic activity. In addition, a number of studies both in vitro and in vivo showed that using chitosan-based NPs could improve biocompatibility and tolerance. ${ }^{16,17}$ So, chitosan has been considered as a promising pharmaceutics material for ophthalmic preparations.

For the purpose of maintaining a constant slow release of $\mathrm{BH}$ from the NP suspensions, montmorillonite (Mt) was considered to be a great potential controlled-release drug delivery vehicle for $\mathrm{BH}$ since it exhibited novel properties, such as large volume-to-mass ratio, flexible interlayer spacing, and excellent cation-exchange capacity. ${ }^{18,19} \mathrm{Mt}$ is a hydrated aluminum silicate with two-dimensional layer structure. Each layer is composed of one aluminum-oxygen octahedral sheet sandwiched between two silicon-oxygen tetrahedral sheets with an interlayer space (about $1 \mathrm{~nm}$ ) to form T-O-T unit cell. ${ }^{20}$ The overall negative surface charge of the $\mathrm{Mt}$ is balanced by the sorption of exchangeable cations $\left(\mathrm{Na}^{+}, \mathrm{Ca}^{2+}\right.$, etc.) in interlayer sites. ${ }^{21}$ Therefore, cationic compounds tend to intercalate into the interlayer space or attach to the surface of Mt to form a complex. It was reported that many drug molecules (such as 5-fluorouracil, ibuprofen and acyclovir) could be successfully intercalated into the interlayer of Mt, leading to a great characteristic for the substance. ${ }^{2-24}$ In our previous study, BH molecules (as cationic compounds) could be successfully intercalated into the interlayer space to form a complex carrier (Mt-BH), which showed significant controlled release. ${ }^{25,26}$

Based on this, we tried to develop a novel ion-exchange controlled-release delivery system to improve bioavailability and compliance of topical ophthalmic preparation for glaucoma treatment. In the present study, with the intercalation of $\mathrm{BH}$ into the interlayer space of the clay, the structure was further integrated with chitosan NPs. Meanwhile, in vitro release performance was investigated, and the irritation was also evaluated to ensure the tolerance for eye. Both in vitro and in vivo experiments were performed to investigate its potential precorneal retention capability. Furthermore, the pharmacokinetics and pharmacodynamics were conducted to evaluate the treatment effects. More importantly, the drug concentration in blood was determined to ensure safety. We hope that the developed BH-Mt/CS NPs could be used as a promising topical ocular drug delivery vehicle with enhanced therapeutic effects and reduced side effects.

\section{Materials and methods Materials}

BH was purchased from Jinan Haohua industry (Shandong, China). CS (Viscosity $\leq 100$ mpa.s, Moisture $\leq 10 \%$ ) was purchased from Jinan Haidebei Marine Bioengineering Co. Ltd. (Shandong, China), with degree of deacetylation $\geq 95 \%$. Mt was obtained from Aladdin (Shanghai, China). 1-(4, 5-dimethylthiazol-2-yl)-3, 5-diphenylformazan (MTT) and all components of buffer solutions were from Sigma (Shanghai, China). The purified water used in all the experiments was obtained from a MilliQ System (Millipore, WA, USA). The organic solvents used in high-performance liquid 
chromatography (HPLC) were of chromatographic grade (Tianjin, China). All the other chemicals and reagents used in the study were of analytical grade. The human immortalized cornea epithelial cells (iHCECs) were kindly provided by Shandong Eye Institute (Qingdao, China), and the iHCEC used in this study were granted approval by the Ethics Committee of Shandong Eye Institute.

\section{Animals}

The experimental rabbits, weighing between 2.0 and $3.0 \mathrm{~kg}$ with no signs of ocular inflammation or gross abnormality, were provided by Guangzhou University of Chinese Medicine Laboratory Animal Center. Animals were individually housed in an air-conditioned and light-controlled room with water and food. All the animals were treated according to the Association for Research in Vision and ophthalmology resolution for the use of animals in research and were approved by the Institutional Animal Care and Use Committee of Guangdong Pharmaceutical University.

\section{Preparation of $\mathrm{BH}-\mathrm{Mt} / \mathrm{CS}$ NPs}

Acid-Mt was formed based on acidification process that has been reported by previous research. ${ }^{25} \mathrm{BH}-\mathrm{Mt} / \mathrm{CS}$ NPs were prepared by ionic gelation of CS with Sodium tripolyphosphate (TPP). ${ }^{27-29}$ Briefly, $5 \mathrm{mg}$ acid-Mt and $61.5 \mathrm{mg} \mathrm{BH}$ were added to $5 \mathrm{~mL}$ acetic acid solution $(2 \% \mathrm{v} / \mathrm{v}, \mathrm{pH} 4.8)$ at $50^{\circ} \mathrm{C}$ for $6 \mathrm{~h}$, and $30 \mathrm{mg}$ chitosan was dissolved in $15 \mathrm{~mL}$ acetic acid solution. The solution containing drug was mixed with completely dissolved chitosan solution. Then, the mixture was ultrasonicated at ice water bath for $5 \mathrm{~min}$. After stirring for $120 \mathrm{~min}, 2.7 \mathrm{~mL}$ of TPP $\left(1.2 \mathrm{mg} \cdot \mathrm{mL}^{-1}\right)$ was added and rapidly ultrasonicated for $5 \mathrm{~s}$; then, the suspension was stirred at $600 \mathrm{rpm} \cdot \mathrm{min}^{-1}$ for $60 \mathrm{~min}$ under room temperature. Finally, mannitol was dispersed into the obtained suspension to adjust the osmotic pressure, ${ }^{30}$ and then, the suspension was conserved at $4^{\circ} \mathrm{C}$.

\section{In vitro characterization of NPs}

\section{Entrapment efficiency and drug-loading rate}

The entrapment efficiency ( $E E \%)$ and drug-loading rate $(D L \%)$ of $\mathrm{BH}-\mathrm{Mt} / \mathrm{CS}$ NPs were determined by dynamic dialysis technique. In brief, $2 \mathrm{~mL}$ of $\mathrm{BH}-\mathrm{Mt} / \mathrm{CS}$ NPs dispersions $\left(2.8 \mathrm{mg} \cdot \mathrm{mL}^{-1}\right)$ were loaded in a dialysis bag with a molecular weight of $12-14 \mathrm{kDa}$ and then immersed in $35 \mathrm{~mL}$ normal saline (NS) with a stirring of $120 \mathrm{rpm} \cdot \mathrm{min}^{-1}$ at $34^{\circ} \mathrm{C}$. At $25 \mathrm{~min}$ (free drug molecules were dialyzed completely), $5 \mathrm{~mL}$ of release medium was withdrawn, and the $\mathrm{BH}$ concentration was determined by HPLC (Agilent 1200, Agilent Technologies, Santa Clara, CA, USA). Ultimate $^{\circledR}$ XB-C18 column (Welch, $4.60 \times 250 \mathrm{~mm}^{2}, 5 \mu \mathrm{m}$ ) was used, and the mobile phase was acetonitrile/trimethylamine (30/70, v/v, pH 3.0). The detector wavelength was $275 \mathrm{~nm}$. The flow rate, column temperature, and injection volume were $1 \mathrm{~mL} \cdot \mathrm{min}^{-1}, 25^{\circ} \mathrm{C}$, and $20 \mu \mathrm{L}$, respectively. The HPLC method was validated with respect to linearity, repeatability, and the limit of quantitation and limit of detection. The $E E \%$ and $D L \%$ of $\mathrm{BH}-\mathrm{Mt} / \mathrm{CS}$ NPs were calculated according to the Equations (1) and (2), respectively:

$$
\begin{aligned}
& \text { Encapsulation efficiency }(E E \%) \\
& =\frac{\text { Total amount of } \mathrm{BH}-\text { Free } \mathrm{BH}}{\text { Total amount of } \mathrm{BH}} \times 100 \% \\
& \text { Drug loading capacity }(D L \%) \\
& =\frac{\text { Total amount of } \mathrm{BH}}{\text { Total weight of } \mathrm{NPs}} \times 100 \%
\end{aligned}
$$

\section{Particle size, zeta potential, and morphology}

Particle size was measured by photon correlation spectroscopy (Beckman Coulter, Brea, CA, USA) at $25^{\circ} \mathrm{C}$ after dilution of formulations with double-distilled water. The average values of three replicates were calculated.

The electrostatic charge on the surface of the particle was measured by Zetasizer (Beckman Coulter) at $25^{\circ} \mathrm{C}$ to predict the physical stability of colloidal suspensions. The measurements were conducted in triplicate.

The morphology was observed by transmission electron microscopy (TEM; Philips TECNAI10, Amsterdam, Holland) at an acceleration voltage of $100 \mathrm{kV}$. After diluting the sample to appropriate concentration with double-distilled water, a drop of the sample was immediately spread on a carbon-coated copper grid and stained with $2 \%$ phosphotungstic acid. Then, the sample was dried at room temperature.

\section{In vitro release studies}

The releases of BH from NPs were studied by dialysis method in artificial tears, which composed of $6.78 \mathrm{~g} \mathrm{NaCl}, 2.18 \mathrm{~g}$ $\mathrm{NaHCO}_{3}, 0.084 \mathrm{~g} \mathrm{CaCl}_{2} \cdot 2 \mathrm{H}_{2} \mathrm{O}$, and $1.38 \mathrm{~g} \mathrm{KCl}$ dissolved in $1,000 \mathrm{~mL}$ deionized water. Two milliliters of sample $\left(2.8 \mathrm{mg} \cdot \mathrm{mL}^{-1}\right)$ was instilled in the dialysis bag and secured with two clamps at each end. The dialysis bag was dipped into the receptor compartment containing $35 \mathrm{~mL}$ of dissolution medium under continuous oscillation at $120 \mathrm{rpm} \cdot \mathrm{min}^{-1}$ at $34^{\circ} \mathrm{C}$. Samples were withdrawn at predefined time intervals and replaced with the same volume of fresh dissolution 
medium to maintain the sink condition. The collected samples were analyzed quantitatively by HPLC.

\section{Irritancy evaluation}

\section{Cell culture and cell viability assay}

Cytotoxicity test was assessed by the mitochondrial-dependent reduction of the tetrazolium salt to formazan. iHCE cell lines were used, which were obtained from Shandong Eye Institute. iHCECs were seeded into 96-well culture plates $\left(1 \times 10^{4}\right.$ cells/well) and were cultured in serum-free medium at $37^{\circ} \mathrm{C}$ in a humidified environment with $5 \% \mathrm{CO}_{2}$ for $24 \mathrm{~h}$. Then, the medium was removed from the wells, and the iHCECs were exposed to different amounts of formulation $(10,20,30,50,80$, and $100 \mu \mathrm{L})$ for $120 \mathrm{~min}$. Subsequently, the medium was carefully sucked out and, MTT solution $\left(5 \mathrm{mg} \cdot \mathrm{mL}^{-1}\right)$ was added to the wells, which were then incubated for $4 \mathrm{~h}$ to obtain blue crystals. After careful removal of the medium, the blue crystals were dissolved with DMSO (150 $\mu \mathrm{L} /$ well). The cell survival rate was quantified by the measurement of optical density at $490 \mathrm{~nm}$ using a microplate reader.

Cell viability was calculated according to formula (3):

$$
\text { Cell viability } \%=\frac{\mathrm{Abs}_{\text {treatment }}}{\mathrm{Abs}_{\text {non-treatment }}} \times 100 \%
$$

\section{Chorioallantoic membrane-trypan blue staining assay} The ocular tolerability of the formulation was investigated by using a chorioallantoic membrane-trypan blue staining (CAM-TBS) test. Briefly, fertilized hen's eggs were incubated for 9 days in adapted circumstance. The portion of egg shell above the air-space was removed and then the shell membrane was peeled off to expose the CAM. Three hundred microliters, of the formulation, was applied onto the CAM for $5 \mathrm{~min}$, and then, the sample was rinsed with distilled water. Subsequently, the CAM was treated with $0.5 \mathrm{~mL}$ of trypan blue solution $\left(1 \mathrm{mg} \cdot \mathrm{mL}^{-1}\right)$ for $1 \mathrm{~min}$. Finally, the dyed CAM was excised and introduced into $1 \mathrm{~mL}$ of formamide for $24 \mathrm{~h}$, and the absorbance of the extract was measured by UV at $611 \mathrm{~nm}$.

\section{Intracellular uptake}

The intracellular uptake of NPs could be monitored by confocal layer scan microscopy (CLSM). Preparations were mounted and examined with a Carl Zeiss LSM310 confocal laser scanning microscope (LEICA TCS SP5II) equipped with a krypton-argon laser. Hoechst33342, curcumin, and DiI were excited with a 346, 425, and $549 \mathrm{~nm}$ emission laser beams, respectively. At first, the NPs were labeled with curcumin (cur-NPs) by substituting BH with curcumin. And they are dialyzed in NS for $24 \mathrm{~h}$ using a dialysis bag (molecular weight cutoff of 8,000-14,000) to remove free curcumin molecules. Then, the iHCECs were incubated with dispersion of cur-NPs in serum-free medium for $4 \mathrm{~h}$. The dispersion was removed, and the cells were washed with PBS several times followed by staining with Hoechst33342 and DiI. The control uptake experiment was iHCEC incubated in serum-free medium. To confirm the intracellular location of cur-NPs, images were captured along the Z-axis, first using the $346 \mathrm{~nm}$ laser for blue hoechst33342 emissions and then the $425 \mathrm{~nm}$ laser for green curcumin emissions, and finally the $549 \mathrm{~nm}$ for red DiI. Merged images were then projected as profiles superimposed with blue, green, and red images.

\section{Precorneal retention}

\section{In vitro precorneal retention}

In vitro precorneal retention was investigated by using in vitro tear-turnover apparatus to simulate the process of generation and elimination of tears as shown in Figure 7A. ${ }^{31}$ Also, multilayered $\mathrm{iHCEC}$ was used as a model to simulate the barrier of corneal epithelial. In brief, the transwell was coated with type I rat-tail collagen $\left(1.5 \mathrm{mg} \cdot \mathrm{mL}^{-1}\right)$ and fibronectin aqueous solution $\left(10 \mu \mathrm{g} \cdot \mathrm{mL}^{-1}\right)$. Then, the fibronectin solution was removed, and the $\mathrm{iHCECs}$ dispersed in the culture medium were seeded onto the polycarbonate filter. After the cells being cultivated submerged in the medium, they were exposed to the air-liquid interface until the iHCECs became multilayered. The transwell in which multilayered iHCECs had been cultured as a turnover chamber. Then, the transwell were placed in a thermostat bath at $34^{\circ} \mathrm{C}$, and a peristaltic pump controlled the inflow and outflow of tears in the chamber. Two hundred and seventy microliters of simulated tear fluid and $30 \mu \mathrm{L}$ of the sample $\left(2.8 \mathrm{mg} \cdot \mathrm{mL}^{-1}\right)$ were translated into the turnover chamber. Two hundred microliters, of solution, was collected every $10 \mathrm{~min}$ and then determined by HPLC with fluorescence detection.

\section{In vivo precorneal retention of $\mathrm{BH}-\mathrm{Mt} / \mathrm{CS}$ NPs}

The precorneal retention ability of $\mathrm{BH}-\mathrm{Mt} / \mathrm{CS}$ NPs was evaluated by using elimination of $\mathrm{BH}$ in tears. ${ }^{32}$ Before the experiment, $1.5 \mathrm{~mL}$ centrifuge tube with filter paper $\left(8 \times 8 \mathrm{~mm}^{2}\right)$ was weighed. The eyes of each group were administered with $100 \mu \mathrm{L}$ sample. At each sampling time, the periopaper was gently inserted into the lower conjunctival sac of the eye for $10 \mathrm{~s}$. The filter paper was then removed and 
immediately put into the centrifuge tubes. The filter paper soaked with tear was weighted, and the weights of pre- and post-sampling were recorded to calculate the amount of tear collected. Two hundred microliters, of methanol, was added to the centrifuge tube. The samples were vortexed and ultrasound, and then, each sample was placed in a refrigerator for $24 \mathrm{~h}$ to extract the drug on the filter paper. With the centrifugation for $15 \mathrm{~min}$ at 15,000 rpm, the supernatant was used to determine the BH concentration via HPLC. The supernatant was determined by HPLC and BH concentration in tears by using following formula:

$$
\mathrm{C}_{0}=\frac{\mathrm{C} \times V_{0} \times \rho}{\Delta \mathrm{m}}
$$

where $\mathrm{C}_{0}$ is the drug concentration in tears, $\mathrm{C}$ is the drug concentration in methanol, $\rho$ is the density of tears $(\rho=1)$, and $\Delta \mathrm{m}$ is the weight of tears.

\section{Aqueous humor pharmacokinetics by microdialysis}

Microdialysis technology is a kind of online sampling analyses, which is removed from the aqueous humor by a diffusion gradient (across the dialysis membrane) established by the continuous perfusion of medium through the probe. ${ }^{33,34}$ In brief, a dose of heparin (100 U $\left.\cdot \mathrm{kg}^{-1}\right)$ was administered through ear vein at $30 \mathrm{~min}$ and $4 \mathrm{~h}$ before surgery to prevent intracameral fibrin formation. Then, the rabbits were anesthetized with pentobarbital sodium $\left(20-30 \mathrm{mg} \cdot \mathrm{kg}^{-1}\right)$ though the rabbit ear marginal vein and the rabbit eyes were instilled two drops of lidocaine eye drop solution to ease the discomfort. Then, the $26 \mathrm{G}$ needle was inserted carefully across the cornea, and the microdialysis probe was immediately introduced through the opening created by the needle. The needle was removed and the active dialysis window totally spanned the anterior chamber. ${ }^{34}$ After 1 day for the aqueous humor to be sufficient for replenishment, the rabbit was administrated formulations. In vivo probe recovery was evaluated by the retrodialysis method. In brief, known concentrations of $\mathrm{BH}$ solution were used as the perfusates with a flow rate of $2.0 \mu \mathrm{L} \cdot \mathrm{min}^{-1}$. All dialysates were collected every $30 \mathrm{~min}$ till $10 \mathrm{~h}$. The microdialysis samples were collected and subjected to HPLC.

\section{Blood pharmacokinetics}

Briefly, the rabbits were kept with full food and water for 2 days and then the rabbits were randomly divided into $\mathrm{BH}$ solution group and BH-Mt/CS NPs group. After the topical application of $100 \mu \mathrm{L}$ formulations into the conjunctival sac of rabbits, the blood samples were collected through the ear margin vein of rabbits at 10, 30, 60, 90, 120, 180, 240, 300 , and $360 \mathrm{~min}$. Acetonitrile (acetonitrile/blood; 2:1) was added to the tube to precipitate the protein in the blood and then vortexed for $2 \mathrm{~min}$. After centrifugation at 12,000 rpm for $10 \mathrm{~min}$, the supernatant was transferred to a fresh tube and evaporated to dryness under a stream of nitrogen. Afterward, the final residue was dissolved in $500 \mu \mathrm{L}$ methanol with vortex-mixing for $1 \mathrm{~min}$ and ultrasonic extraction for $30 \mathrm{~min}$. The sample was filtrated through $0.22 \mu \mathrm{m}$ membrane. Finally, the filtered solution was injected into the HPLC system for analysis.

\section{Pharmacodynamics}

New Zealand albino rabbits were used as model animals. Rabbits were examined at the beginning of the experiment to ensure the rabbit eye is without ocular inflammation and other diseases. After general anesthesia with 3\% pentobarbital sodium (about $30 \mathrm{mg} \cdot \mathrm{kg}^{-1}$ ), local anesthetic with one drop of $0.2 \%(\mathrm{w} / \mathrm{v})$ lidocaine hydrochloride was instilled into the eye. The IOP readings were monitored by an indentation tonometer (YZ7A, Suzhou Visual Technology Co., Ltd., Suzhou, China). Then, the ocular hypertension model was induced by filling the stomach with NS $\left(100 \mathrm{~mL} \cdot \mathrm{kg}^{-1}\right)$. Immediately, $100 \mu \mathrm{L}$ of BH-Mt/CS NPs and BH solution were dripped into the conjunctival sac of left eye (treatment eye). And then, the eye was kept open for about $1 \mathrm{~min}$ to prevent the eye drops from overflowing. To avoid experimental bias, another eye was instilled with $100 \mu \mathrm{L}$ NS (as a control). IOP was measured in rabbits at 15, 30, 45, 60, 90, 120, 180, 240, 300, and $360 \mathrm{~min}$ after single dosing. All measurements were made by the same operator unaware of the treatment. Change in IOP $(\Delta \mathrm{IOP})$ was expressed as follows: $\Delta \mathrm{IOP}=\mathrm{IOP}_{\text {control eye }}-\mathrm{IOP}_{\text {treatment eye }}$.

\section{Results and discussion Preparation of $\mathrm{BH}-\mathrm{Mt} / \mathrm{CS}$ NPs}

For this study, chitosan NPs were prepared by ionotropic gelation with the addition of TPP to chitosan solution under a moderate temperature without using organic solvents (toxic substance) and formaldehyde (carcinogenic substance) as the crosslinking curing agents. The formation of $\mathrm{BH}-\mathrm{Mt} / \mathrm{CS}$ NPs is shown in Figure 1.

As shown in Table 1, the particle size of BH-Mt/CS NPs and Mt/CS NPs (without BH) were $460 \pm 0.6$ and $423 \pm 0.9 \mathrm{~nm}$, respectively, and the diameter distributions of the NPs were relatively narrow (about 0.2 ; Figure $2 \mathrm{~A}$ and $\mathrm{B}$ shows the 


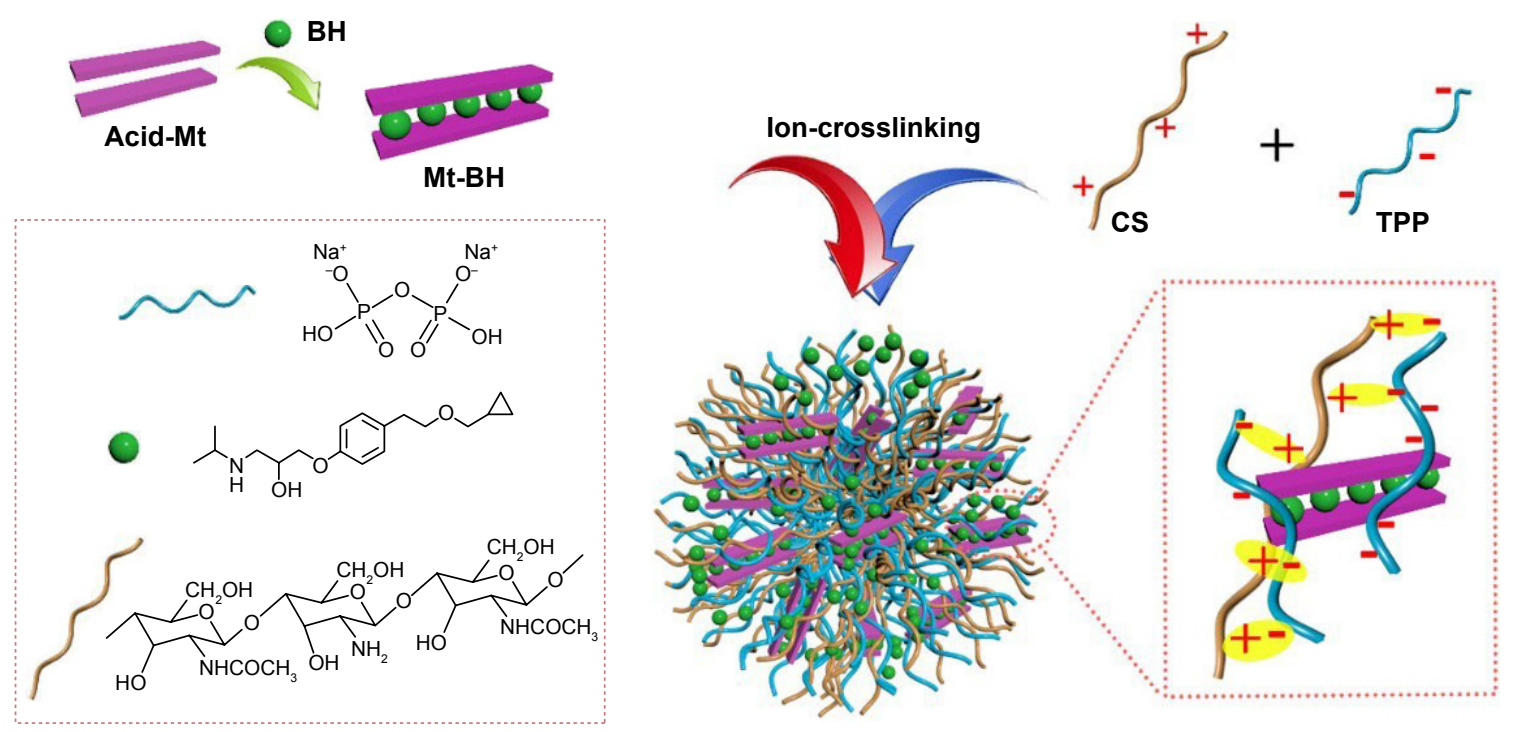

Figure I A schematic of the preparation process of $\mathrm{BH}-\mathrm{Mt} / \mathrm{CS} \mathrm{NPs}$.

Abbreviations: $\mathrm{BH}$, betaxolol hydrochloride; CS, chitosan; Mt, montmorillonite; NPs, nanoparticles; TPP, tripolyphosphate.

particle size distributions of BH-Mt/CS NPs and Mt/CS NPs, respectively). These properties may be advantageous for the uptake of NPs through the cornea.$^{35}$ The encapsulation efficiency and drug-loading capacity of BH-Mt/CS NPs were $36.13 \% \pm 0.16 \%$ and $14.5 \% \pm 0.06 \%$, respectively. From the results of Zeta potential, it was found that the surfaces of two kinds of CS NPs have positive charges of about $+29 \pm 0.18 \mathrm{mV}$, which could offer effective electric repulsion to avoid the aggregation of particles. Also, the positively charged surface of CS NPs was mainly due to the cationic characteristic of CS. This special characteristic enables it to interact with negatively charged mucosal mucin in preocular site, which may be beneficial to improve preocular retention time for ophthalmic drug delivery.

A representative TEM image of $\mathrm{BH}-\mathrm{Mt} / \mathrm{CS}$ NPs is presented in Figure 3. One can see that the tested NPs were predominantly spherical in the shape with an irregular surface. The average diameter of NPs was about $450 \mathrm{~nm}$, which was smaller than the diameter determined by photon correlation spectroscopy (dynamic light scattering). This could be explained by the following: the freshly prepared NPs

Table I The particle size, zeta potential, encapsulation efficiency, and loading capacity of Mt-BH/CS NPs and Blank CS NPs (mean \pm SD)

\begin{tabular}{llllll}
\hline Formulation & $\begin{array}{l}\text { Particle } \\
\text { size/nm }\end{array}$ & $\begin{array}{l}\text { Zeta } \\
\text { potential/mV }\end{array}$ & PDI & EE/\% & DL/\% \\
\hline NPs & $460 \pm 0.6$ & $29 \pm 0.18$ & 0.276 & $36.13 \pm 0.16$ & $14.5 \pm 0.06$ \\
Blank NPs & $423 \pm 0.9$ & $27 \pm 0.62$ & 0.26 & & \\
\hline
\end{tabular}

Abbreviations: $\mathrm{BH}$, betaxolol hydrochloride; CS, chitosan; DL, drug loading capacity; Mt, montmorillonite; NPs, nanoparticles; EE, entrapment efficiency; PDI, polydispersity index. suspensions were used for the detection of photon correlation spectroscopy, and the chitosan and Mt can swell under acetic acid aqueous solution; whereas, the sample needs to be dry before the TEM experiments. Therefore, the diameter of dry sample was smaller than that of swelled sample.

\section{In vitro release studies}

It is essential to evaluate the in vitro release characteristics in artificial tears to forecast the in vivo performance of BH-Mt/ CS NPs. In vitro release study of BH solution, BH-CS NPs (without $\mathrm{Mt}$ ), BH-Mt/CS NPs, and BH-Mt formulations during $10 \mathrm{~h}$ are shown in Figure 4. BH solution formulation showed a significantly fast release profile with about $100 \%$ within $2 \mathrm{~h}$ due to its low molecular weight and water solubility of BH molecules. It was found that BH-Mt complex (Figure 4d) showed slower release profiles, with about $100 \%$ within $3.5 \mathrm{~h}$, than $\mathrm{BH}$ solution, due to ion-exchange process of $\mathrm{BH}$ from $\mathrm{Mt}$ interlayer and adsorption of $\mathrm{BH}$ on the surface of Mt. Also, two NPs formulations containing chitosan (Figure 4a and b) displayed an initial burst release and then a sustained release for $10 \mathrm{~h}$ without a fast burst release. Drug adsorption on the surface of the NPs could have been responsible for the presence of initial burst during drug release. ${ }^{36}$ The BH-Mt/CS NPs (with Mt) was slower than BH-CS NPs (without Mt), owing to their high cationexchange capacity and tortuous path in swelling of the NPs. Meanwhile, it was found that BH-Mt composite was slower than BH-CS NPs, which may be attributed to the strong bond between the positively charged $\mathrm{BH}$ and the negatively charged Mt layer, making $\mathrm{BH}$ attain a very slow release. 

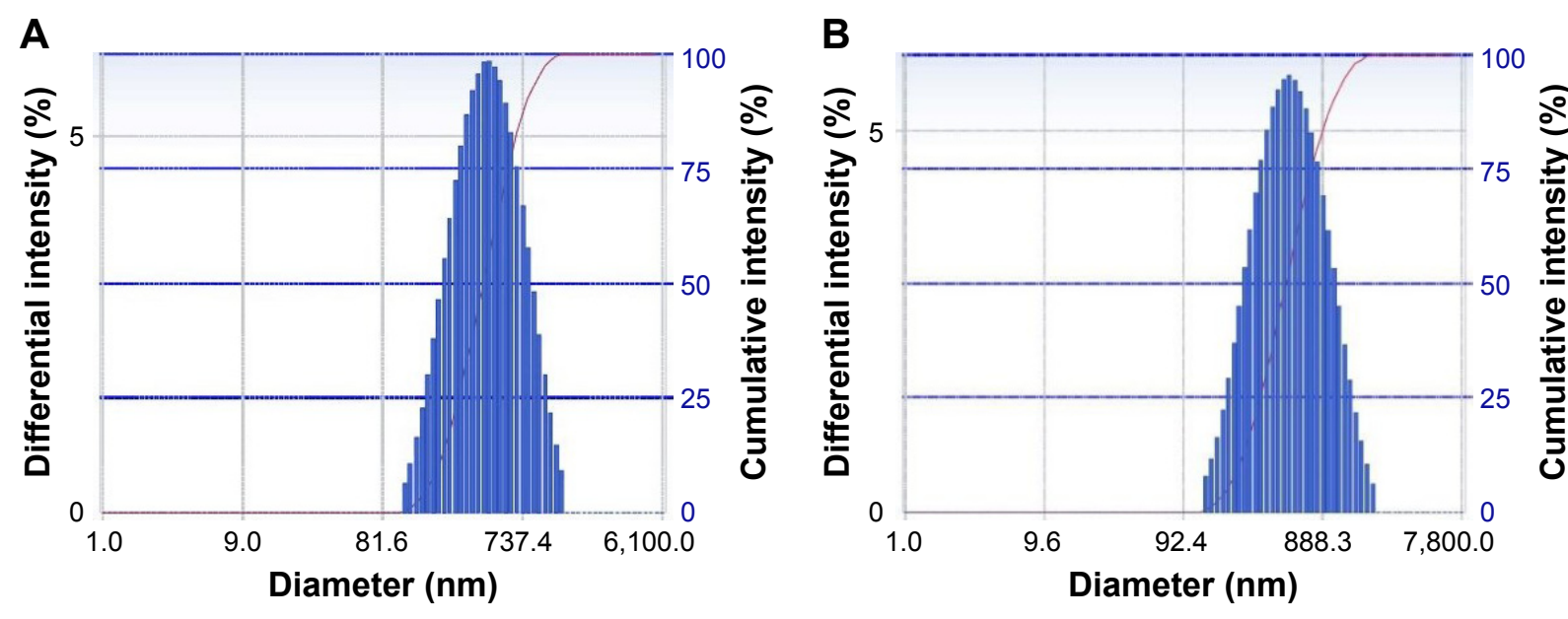

Figure 2 The particle size distributions of (A) BH-Mt/CS NPs and (B) Mt/CS NPs. Note: The particle size was measured by DLS.

Abbreviations: $\mathrm{BH}$, betaxolol hydrochloride; CS, chitosan; DLS, dynamic light scattering; Mt, montmorillonite; NPs, nanoparticles.

Kinetically, the release of BH from NPs appeared to follow Ritger-Pappa equation, which suggested the controlledrelease mechanism of diffusion and erosion. It has been proposed that controlled drug release from NPs is important to increase the bioavailability and therapeutic effect, since it can prolong the residence time of the drug on the eye surface, thus avoiding immediate removal of the drug from the corneal surface by the flow of tears.

\section{Irritation evaluation}

\section{Cytotoxicity}

The instillation of topical eye drops could directly contact with the surface of epithelia cells, which might cause cytotoxic effects. For this reason, the ocular tolerability of BH-Mt/ CS NPs should be taken into consideration before in vivo

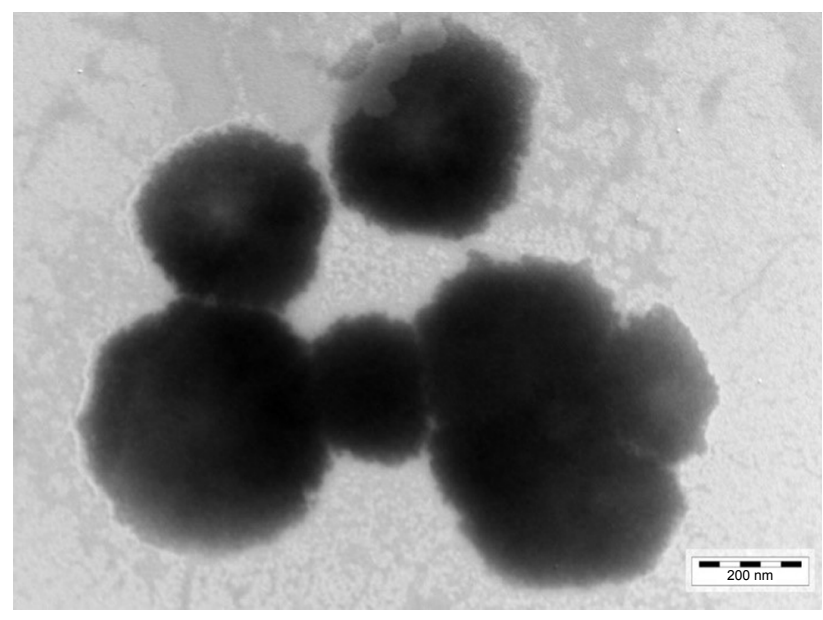

Figure 3 TEM image of the Mt-BH/CS NPs.

Abbreviations: $\mathrm{BH}$, betaxolol hydrochloride; $\mathrm{CS}$, chitosan; Mt, montmorillonite; NPs, nanoparticles; TEM, transmission electron microscopy. application. In this study, iHCEC was used as a substitute for human and animal corneal epithelial in eye irritation testing, which could reduce the use of animals to a large extent.

After being exposed to BH solution, Mt/CS NPs, and BH-Mt/CS NPs, the cell viability of iHCEC is shown in Figure 5A. In general, the viability of iHCEC was decreased by increasing the concentration of the samples as well as the incubation time. For BH solution, it could be found that the cell viability was $>60 \%$ when the administration of volume is $<20 \mu \mathrm{L}$. However, when the drug concentration is $>50 \mu \mathrm{L}$, the cell survival rate fell to $<20 \%$, suggesting that $\mathrm{BH}$ had a toxic effect on iHCEC in higher concentration levels. The cell viability of Mt/CS NPs was $>80 \%$ at the amount of $50 \mu \mathrm{L}$ for 120 min implying that the new ocular carrier

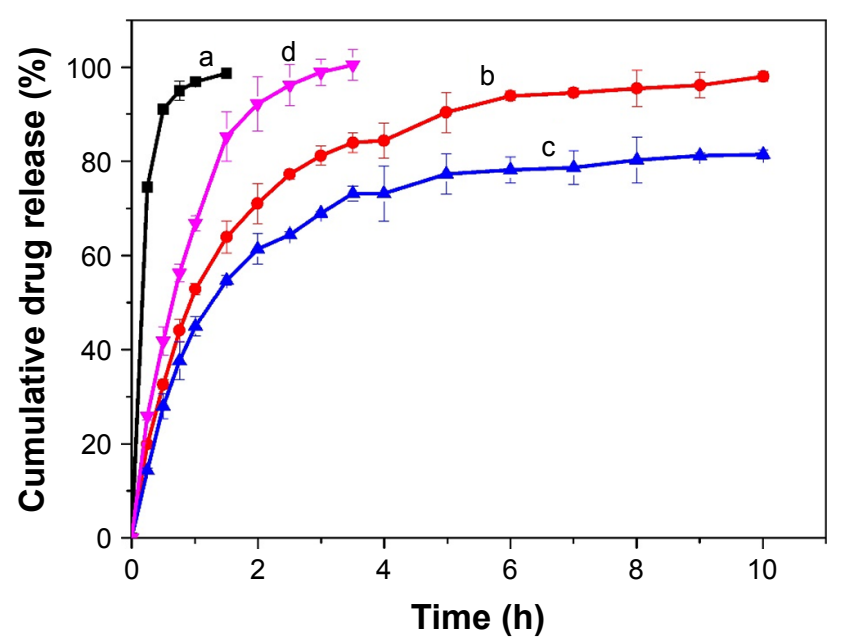

Figure 4 In vitro dialysis release study of $\mathrm{BH}$ from formulations: (a) $\mathrm{BH}$ solution, (b) BH-CS NPs, (c) BH-Mt/CS NPs, and (d) BH-Mt. The cumulative drug release (\%) is plotted against time $(\mathrm{h})$.

Abbreviations: $\mathrm{BH}$, betaxolol hydrochloride; $\mathrm{CS}$, chitosan; Mt, montmorillonite; NPs, nanoparticles. 

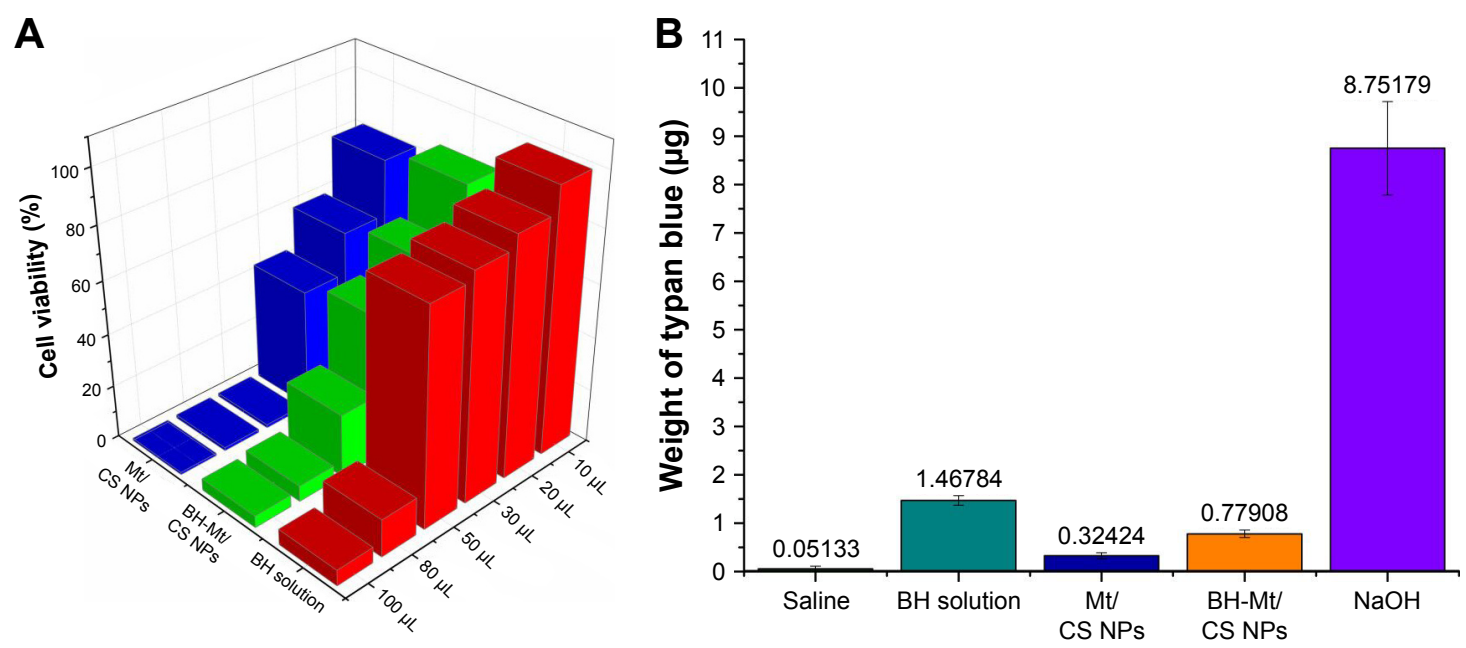

Figure 5 (A) Percent cell viability of iHCEC cells determined by MTT assay after exposing them to different amounts $(10,20,30,50,80$, and I $00 \mu \mathrm{L})$ of $B H$ solution, $\mathrm{Mt} / \mathrm{CS}$ $\mathrm{NPs}$, and $\mathrm{BH}-\mathrm{Mt} / \mathrm{CS} \mathrm{NPs}$ for $120 \mathrm{~min}$. (B) Eye irritancy induced by different concentrations of BH solution, Mt/CS NPs, and BH-Mt/CS NPs determined by the in vitro HET-CAM method. $\mathrm{NaOH}$ was used as a positive control and normal saline as a negative control, respectively.

Abbreviations: BH, betaxolol hydrochloride; CS, chitosan; HET-CAM, Hen's Egg Test Chorioallantoic Membrane; Mt, montmorillonite; NPs, nanoparticles; iHCEC, human immortalized cornea epithelial cells.

was innoxious for iHCEC due to excellent properties of chitosan (such as biocompatibility, nontoxicity, good ocular tolerance, and great wound healing). ${ }^{37-39}$ The cell viability of BH-Mt/CS NPs was $>50 \%$ at the amount of $30 \mu \mathrm{L}$, which showed greater cytotoxicity than that of Mt/CS NPs. It may be attributed to the great stimulation on the cells of free drug in NPs suspensions. In comparison with BH solution, the cytotoxicity of Mt-BH/CS NPs was lower, which may be explained by the fact that chitosan-based NPs can protect the cells from damaging by BH (raw drug).

\section{Chorioallantoic membrane-trypan blue staining}

The CAM of the chick embryo is a complete tissue including veins, arteries, and capillaries, which could respond to injury caused by some chemical substance (similar to the conjunctival tissue of rabbit eyes). This could avoid conflict with the ethical and legal obligations. Hagino et al developed an objective evaluation technique using the amount of trypan blue absorbed at the site of treatment as an indicator of injury to the CAM. ${ }^{40}$ Lagarto et al have also observed a good correlation $(\mathrm{r}=0.835 ; p<0.0001)$ between the amount of dye absorbed by the CAM and the Draize eye irritation test score, suggesting that CAM-TBS may be suitable for predicting corneal injury. ${ }^{41}$ Meanwhile, the CAM-TBS assay is inexpensive and simple. More importantly, it can provide an in vitro alternative method to predict the damage of the ocular structures caused by chemical substances or cosmetics. Hence, it is a promising alternative method to substitute the Draize eye irritation test.

The developed formulation was checked by using this method, and the result was compared with NS and $\mathrm{NaOH}$, which were used as negative control and positive control, respectively. The absorption amount of trypan blue was in the following order: $\mathrm{NaOH}$ (positive control) $>>\mathrm{BH}$ solution $>\mathrm{Mt}-\mathrm{BH} / \mathrm{CS} \mathrm{NPs}>\mathrm{Mt} / \mathrm{CS} \mathrm{NPs}>\mathrm{NS}$ (negative control; Figure $5 \mathrm{~B}$ ). These results were consistent with the value estimated by the cytotoxicity, which revealed that Mt-BH/ CS NPs were safe for ocular drug delivery.

\section{Cellular uptake}

The cellular uptake of Mt/CS NPs was detected by CLSM. According to Figure 6, it was found that iHCEC cells of control group showed normal morphological details as shown in Figure 6B and C (cell nucleus staining with blue fluorescence and cytoskeletal staining with red fluorescence). When exposed to the culture medium alone, no green fluorescence was detected in control group. However, after exposed to $\mathrm{Mt} / \mathrm{CS} \mathrm{NPs}$ for $4 \mathrm{~h}$, the iHCEC cells had high green fluorescent inside them (Figure 6d), and the nucleus and cytoskeletal showed no alternation of morphology compared to the control cell (Figure $6 \mathrm{~b}$ and $\mathrm{c}$ ), which could be explained by the fact that Mt/CS NPs were harmless to the cells. Other evidences of the safe application of NPs to the ocular surface have been reported. ${ }^{2}$ Furthermore, it was clearly seen that green fluorescence was detected only in cytoskeletal, indicating the uptake into cytoskeletal.

\section{Precorneal residence time}

\section{In vitro precorneal residence}

Figure 7B shows the plot of $\mathrm{BH}$ concentration versus time profiles after the instillation of formulations. The elimination of $\mathrm{BH}$ solution was found to be much faster than that of the 

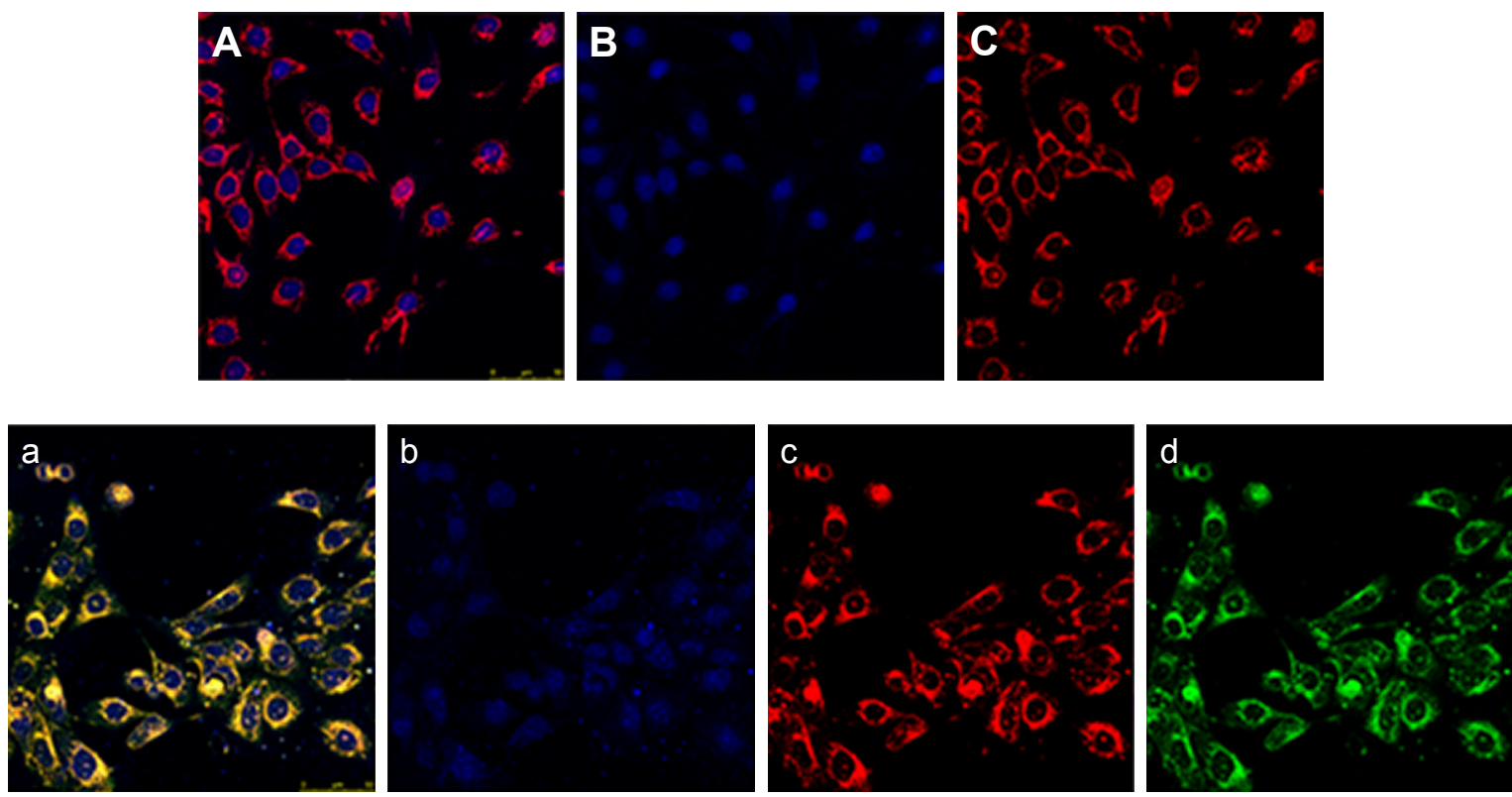

Figure 6 Fluorescence image of immortalized human corneal epithelial cells after incubation with culture medium ([A] merged images with blue, green, and red image; [B] cell nucleus staining with blue fluorescence; [C] cytoskeletal staining with red fluorescence) and Mt/CS NPs ([a] merged images with blue, green, and red image; [b] cell nucleus staining with blue fluorescence; [c] cytoskeletal staining with red fluorescence; and [d] Mt/CS NPs staining with green fluorescence) for $4 \mathrm{~h}$.

Abbreviations: CS, chitosan; Mt, montmorillonite; NPs, nanoparticles.

Mt-BH/CS NPs. The drug concentration of BH solution could not be detected after $80 \mathrm{~min}$. However, Mt-BH/CS NPs formulation was low but still detectable, and drug could remain detectable up to $120 \mathrm{~min}$ on the cell surface, which was a significant increase in residence time. The longer retention time was attributed to the enhanced mucoadhesive properties of the Mt-BH/CS NPs formulation (Figure 7C) and the sustained release of $\mathrm{BH}$ from Mt-BH/CS NPs. The enhanced mucoadhesive properties may provide an intimate contact between drugs and ocular surface tissues, and the sustained release could avoid the BH being quickly removed, thus, reducing the elimination of BH. Its potential prolonged retention is expected to increase the bioavailability of ophthalmic drugs, which needs to be demonstrated by further work.

\section{In vivo precorneal residence}

It is proposed that ocular therapy would be significantly improved if the precorneal residence time of drug could be increased. Herein, this profile was designed to evaluate the precorneal retention capability of BH-Mt/CS NPs, which would be beneficial for predicting the bioavailability of the drug for effective treatment of glaucoma. ${ }^{42}$ After applying
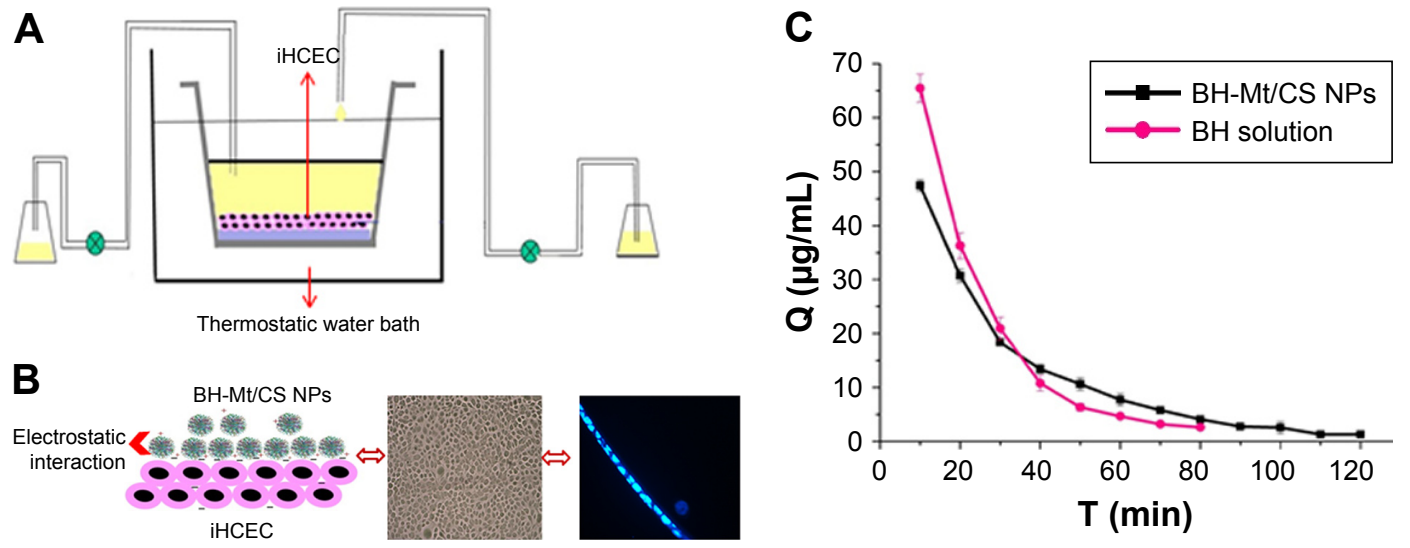

Figure 7 (A) Schematic diagram of the in vitro tear-turnover apparatus. The model incorporates an insert containing iHCEC as a turnover chamber; the external basal side of the insert was sealed to avoid a down diffusion of material. The temperature of thermostat bath is $34^{\circ} \mathrm{C}$ (the temperature of human tear film). Two peristaltic pumps were used to control the inflow and outflow of simulated tears in the chamber. (B) Schematic diagram of the BH-Mt/CS NPs with positive charge could retain on the iHCEC (negative charge) by electrostatic interaction. (C) BH concentration in the cornea/tear film compartment after the instillation of $\mathrm{BH}$ solution and $\mathrm{BH}-\mathrm{Mt} / \mathrm{CS} \mathrm{NPs}$. Abbreviations: $\mathrm{BH}$, betaxolol hydrochloride; CS, chitosan; Mt, montmorillonite; NPs, nanoparticles; iHCEC, human immortalized cornea epithelial cells. 
$\mathrm{BH}$ solution and $\mathrm{BH}-\mathrm{Mt} / \mathrm{CS}$ NPs $\left(2.8 \mathrm{mg} \cdot \mathrm{mL}^{-1}\right)$ to rabbit eye, drainage and elimination processes started. In the first few minutes, the $\mathrm{BH}$ tear film concentration was high after the instillation. But a rapid decrease trend was observed after $10 \mathrm{~min}$ due to the mechanical elimination of the excess instilled volume (Figure 8). After the initial rapid decrease, the proceeds of $\mathrm{BH}$ elimination mainly depend on normal tear turnover, leakage of nasolacrimal duct, and tear dilution.

It was obvious that the $\mathrm{BH}$ concentration of $\mathrm{BH}-\mathrm{Mt} / \mathrm{CS}$ NPs was higher than that of $\mathrm{BH}$ solution at each time point, and the maximum concentration $\left(\mathrm{C}_{\max }\right)$ of $474.86 \mu \mathrm{g} \cdot \mathrm{mL}^{-1}$ was measured at $15 \mathrm{~min}$ after eye drop administration which is 1.70-fold than that of $\mathrm{BH}$ solution. In addition, the $\mathrm{BH}$ concentration of $\mathrm{BH}$ solution in tears cannot be detected at $90 \mathrm{~min}$, and the $\mathrm{BH}$ concentration of BH-Mt/CS NPs was detected even at $240 \mathrm{~min}$. This might be ascribed to positively charged NPs having a strong affinity to the negatively charged corneal surface. Meanwhile, the presence of free $\mathrm{NH}_{2}$ and $\mathrm{OH}$ groups of $\mathrm{CS}$ molecules could form hydrogen bonds with the mucin of corneal surface. BH-Mt/CS NPs showed the highest $\mathrm{BH}$ levels at every time point, reducing much drug loss from the precorneal, which could be attributed to the good bioadhesion and low irritant. This result indicated that the BH-Mt/CS NPs might prolong the retention time of the drugs on the ocular surface and slow down the drug elimination, thus enhancing ocular bioavailability of topically dosed ophthalmic drugs. Consequently, it may decrease the dosing frequency for patients, and finally much lower dose of $\mathrm{BH}$ is required to achieve the desired therapeutic levels in comparison with $\mathrm{BH}$ solution therapy.

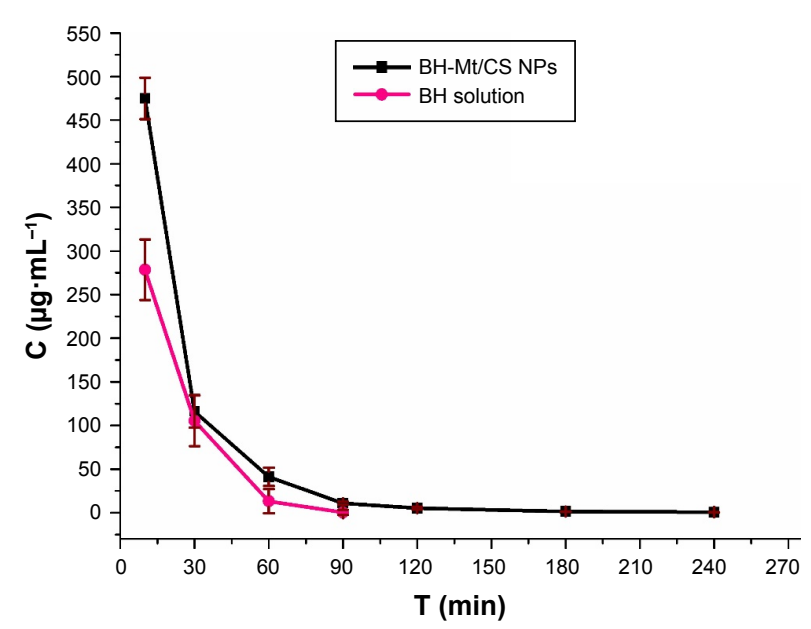

Figure 8 Tear fluid concentration-time curve after topical application of $\mathrm{BH}$ solution and $\mathrm{BH}-\mathrm{Mt} / \mathrm{CS} \mathrm{NPs}$ in the rabbit eyes.

Abbreviations: $\mathrm{BH}$, betaxolol hydrochloride; $\mathrm{CS}$, chitosan; Mt, montmorillonite; NPs, nanoparticles.

\section{Aqueous humor pharmacokinetics through microdialysis}

The major problem for ophthalmic drug delivery is the rapid and extensive precorneal loss caused by drainage and high tear turnover. For this, maintaining adequate drug accumulation in cornea or aqueous humor plays an important key role for a successful therapy. Therefore, it is important to determine the drug concentration in aqueous humor. ${ }^{9}$ Microdialysis sampling online technology was utilized to evaluate the pharmacokinetics in the aqueous humor. The in vivo recovery was evaluated to ensure that the implanted probes were sufficiently functional. The results showed that the in vivo recovery rate was $28.90 \%$. The $\mathrm{BH}$ concentrations in the rabbit aqueous humor of $\mathrm{BH}-\mathrm{Mt} / \mathrm{CS}$ NPs and BH solution are shown in Figure 9. For BH solution, the maximum $\mathrm{BH}$ concentration $\left(\mathrm{C}_{\max }\right)$ of $\mathrm{BH}$ solution $\left(7.05 \mu \mathrm{g} \cdot \mathrm{mL}^{-1}\right)$ was quickly attained after $30 \mathrm{~min}$ of administration. However, it rapidly fell to $1.39 \mu \mathrm{g} \cdot \mathrm{mL}^{-1}$ and then it reached undetectable values in $300 \mathrm{~min}$. For the BH-Mt/ CS NPs, it attained the peak concentration $\left(7.20 \mu \mathrm{g} \cdot \mathrm{mL}^{-1}\right)$ at $120 \mathrm{~min}$ and followed a slow downtrend. It was obvious that the drug concentration of BH-Mt/CS NPs could maintain a certain level in the aqueous humor for $600 \mathrm{~min}$. The pharmacokinetic parameters of the two formulations are listed in Table 1. The area under the curve (AUC) and mean residence time of $\mathrm{BH}-\mathrm{Mt} / \mathrm{CS}$ NPs were $\sim 1.99$ - and 1.75-fold higher than that of $\mathrm{BH}$ solution. This result indicated that BH-Mt/CS NPs could significantly prolong the residence time and increase the ocular bioavailability of $\mathrm{BH}$ in comparison with $\mathrm{BH}$ solution.

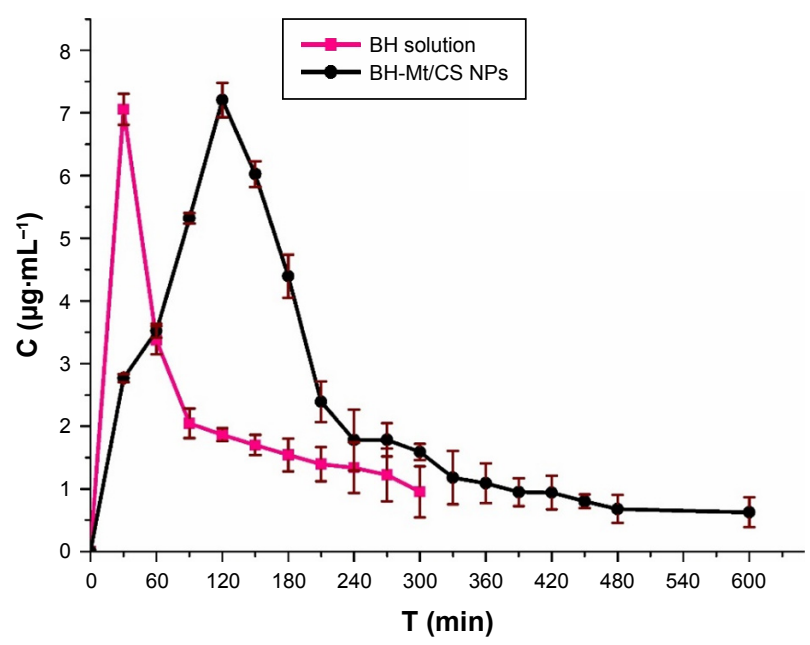

Figure 9 Concentration of $\mathrm{BH}$ in rabbit aqueous humor after instillation of the $\mathrm{BH}$ solution and $\mathrm{BH}-\mathrm{Mt} / \mathrm{CS}$ NPs.

Abbreviations: $\mathrm{BH}$, betaxolol hydrochloride; $\mathrm{CS}$, chitosan; Mt, montmorillonite; NPs, nanoparticles. 


\section{The $\mathrm{BH}$ concentration in bloodstream}

Topically applied ophthalmic drugs are designed to enter some internal ocular structure to exert some local effect. However, a part of drug was distributed to other areas of the body (particularly into the general systemic circulation) after the topical administration, resulting in toxic side effects, such as bradycardia, dizziness and sleepwalking, rhinitis and dysuria. It could be especially harmful to both pediatric and geriatric patients because these groups may be less able to tolerate large doses caused by systematical absorption. ${ }^{43}$ In this study, the BH concentration in blood was investigated and the concentration-time profile of $\mathrm{BH}$ in blood after topical dose in conscious rabbits is shown in Figure 10. These two formulations could enter blood due to high permeability of $\mathrm{BH}$ molecules. The $\mathrm{BH}$ concentration of BH solution sample in blood was nearly twofold higher than that of BH-Mt/CS NPs within 60 min after the administration. In addition, the $\mathrm{BH}$ concentration of BH-Mt/CS NPs maintained a level of nearly $0.07 \mu \mathrm{g} \cdot \mathrm{mL}^{-1}$ only with slight fluctuation. The results indicated that BH-Mt/CS NPs have lower $\mathrm{BH}$ concentration in bloodstream than that of $\mathrm{BH}$ solution, which might minimize potential risk of side effects.

\section{IOP-lowering effect on rabbits}

The pharmacological response (the decrease in IOP, $\Delta \mathrm{IOP}$ ) versus time profiles for $\mathrm{BH}$ solution and $\mathrm{BH}-\mathrm{Mt} / \mathrm{CS}$ NPs is presented in Figure 11. It could be clearly seen that the administration of the two formulations showed a significant IOP-lowering effect. BH solution exhibited a significant IOP-lowering effect within 30-90 min, and then, the effect became mild, possibly due to the quick release of

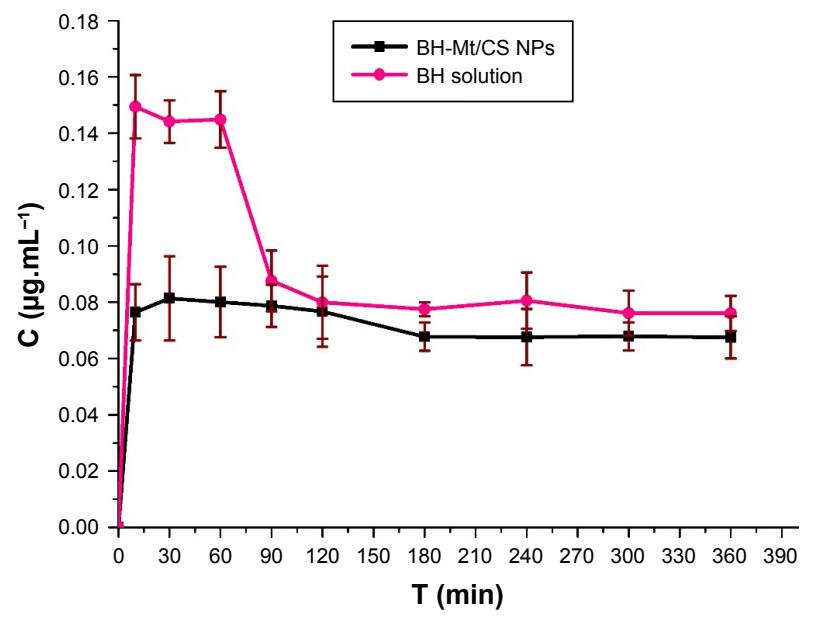

Figure 10 The concentration of $\mathrm{BH}$ in blood after instillation of the $\mathrm{BH}$ solution and $\mathrm{BH}-\mathrm{Mt} / \mathrm{CS} \mathrm{NPs}$.

Abbreviations: $\mathrm{BH}$, betaxolol hydrochloride; $\mathrm{CS}$, chitosan; Mt, montmorillonite; NPs, nanoparticles.

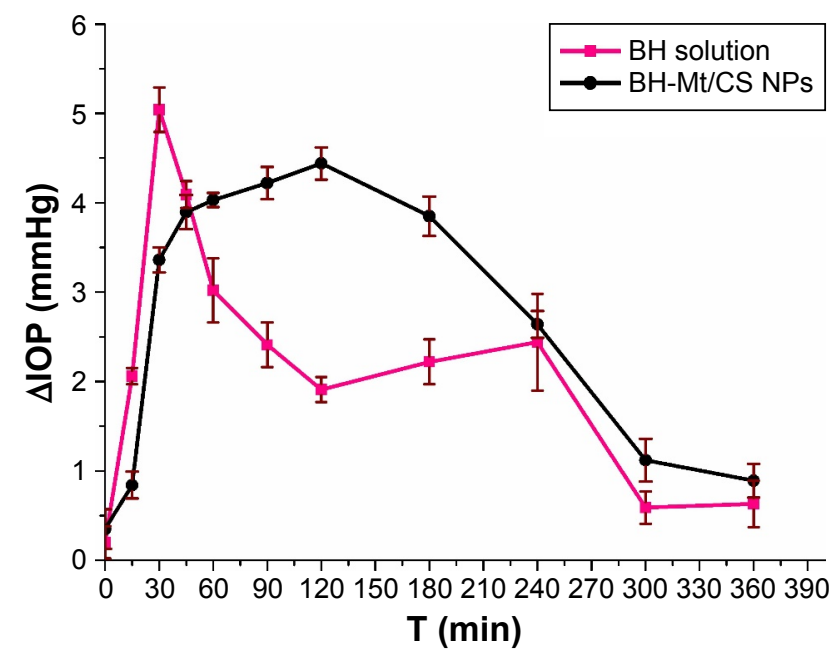

Figure II The pharmacological response (the decrease in IOP, $\triangle \mathrm{IOP}$ ) versus time profiles for $\mathrm{BH}$ solution and $\mathrm{BH}-\mathrm{Mt} / \mathrm{CS}$ NPs.

Abbreviations: $\mathrm{BH}$, betaxolol hydrochloride; $\mathrm{CS}$, chitosan; IOP, intraocular pressure; Mt, montmorillonite; NPs, nanoparticles.

drug molecules for $\mathrm{BH}$ solution, and then eliminated from precorneal. Although the strongest IOP-lowering effect of BH-Mt/CS NPs (4.44 mmHg) was lower than that of BH solution $(5.04 \mathrm{mmHg})$, the IOP-lowering effect of BH-Mt/ CS NPs could maintain slow, sustained downward trend to $360 \mathrm{~min}$. This could be attributed to the NPs releasing the drug in a sustained manner and remaining on the preocular surface for a longer time. These results showed that BH-Mt/ CS NPs could make a significant decrease of IOP and produce a therapeutic effect on glaucomatous rabbits.

\section{Conclusion}

In summary, a novel kind of ion-exchange drug delivery system was successfully prepared to overcome the conundrum of ocular drug delivery. The ionic interaction between the negatively charged TPP and the positively charged CS drive the formation of this novel carrier. It was clearly observed that the cumulative release (\%) was extended with the presence of Mt, which produced a tortuous path in swelling of the NPs. ${ }^{1}$ The irritation test on iHCEC and CAM-TBS test showed no obvious toxicity. It was exciting that both in vitro and in vivo precornea retention studies showed extended precorneal retention time due to the tight contact with the negatively charged cornea mucin resulting from the positive charge $(+29 \pm 0.18 \mathrm{mV})$. Pharmacokinetic in aqueous humor showed greater AUC than BH solution, suggesting improvement in drug residence time and higher bioavailability of drug. Pharmacodynamic results found that BH-Mt/CS NPs have a significant reduction in IOP with longer action duration. In addition, the drug concentration in 
blood was lower in comparison with $\mathrm{BH}$ solution, suggesting lower side effect. We believe that the obtained BH-Mt/CS NPs may present new possibilities to create a class of nanocarrier suitable for the glaucoma treatment to get better patient compliance and good therapeutic results.

\section{Acknowledgments}

This work was financially supported by the National Natural Science Foundation of China (Grant No 51102052), Medical Scientific Research Foundation of Guangdong Province, China (Grant No A2016275), and Science and Technology Planning Project of Guangdong Province, China (Grant No 2017B030314175). Guangdong Provincial Key Laboratory of Mineral Physics and Materials and Guangdong Provincial Engineering Center of Topical Precise Drug Delivery System are acknowledged for their help in performing the experiments. The eye institute of Shandong province in China is acknowledged for the provision of human immortalized cornea epithelial cell line (iHCEC).

\section{Disclosure}

The authors report no conflicts of interest in this work.

\section{References}

1. Peng CC, Burke MT, Carbia BE, Plummer C, Chauhan A. Extended drug delivery by contact lenses for glaucoma therapy.J Control Release. 2012;162(1):152-158.

2. Katiyar S, Pandit J, Mondal RS, et al. In situ gelling dorzolamide loaded chitosan nanoparticles for the treatment of glaucoma. Carbohydr Polym. 2014;102:117-124.

3. Jung HJ, Abou-Jaoude M, Carbia BE, Plummer C, Chauhan A. Glaucoma therapy by extended release of timolol from nanoparticle loaded silicone-hydrogel contact lenses. J Control Release. 2013;165(1): 82-89.

4. Huang W, Zhang N, Hua H, et al. Preparation, pharmacokinetics and pharmacodynamics of ophthalmic thermosensitive in situ hydrogel of betaxolol hydrochloride. Biomed Pharmacother. 2016;83:107-113.

5. Chhonker YS, Prasad YD, Chandasana H, et al. Amphotericin-B entrapped lecithin/chitosan nanoparticles for prolonged ocular application. Int J Biol Macromol. 2015;72:1451-1458.

6. Leonardi A, Bucolo C, Drago F, Salomone S, Pignatello R. Cationic solid lipid nanoparticles enhance ocular hypotensive effect of melatonin in rabbit. Int J Pharm. 2015;478(1):180-186.

7. Baig MS, Ahad A, Aslam M, Imam SS, Aqil M, Ali A. Application of Box-Behnken design for preparation of levofloxacin-loaded stearic acid solid lipid nanoparticles for ocular delivery: optimization, in vitro release, ocular tolerance, and antibacterial activity. Int J Biol Macrom. 2016;85:258-270.

8. Dillen K, Vandervoort J, Van den Mooter G, Ludwig A. Evaluation of ciprofloxacin-loaded Eudragit RS100 or RL100/PLGA nanoparticles. Int J Pharm. 2006;314(1):72-82.

9. Ustundag-Okur N, Gokce EH, Bozbiyik DI, Egrilmez S, Ozer O, Ertan G. Preparation and in vitro-in vivo evaluation of ofloxacin loaded ophthalmic nano structured lipid carriers modified with chitosan oligosaccharide lactate for the treatment of bacterial keratitis. Eur J Pharm Sci. 2014;63:204-215.

10. Battaglia L, Serpe L, Foglietta F, et al. Application of lipid nanoparticles to ocular drug delivery. Expert Opin Drug Deliv. 2016;13(12): $1743-1757$.
11. Giarmoukakis A, Labiris G, Sideroudi H, et al. Biodegradable nanoparticles for controlled subconjunctival delivery of latanoprost acid: in vitro and in vivo evaluation. Preliminary results. Exp Eye Res. 2013; 112:29-36.

12. Fangueiro JF, Andreani T, Egea MA, et al. Design of cationic lipid nanoparticles for ocular delivery: development, characterization and cytotoxicity. Int J Pharm. 2014;461(1-2):64-73.

13. Park CG, Kim YK, Kim MJ, et al. Mucoadhesive microparticles with a nanostructured surface for enhanced bioavailability of glaucoma drug. $J$ Control Release. 2015;220(Pt A):180-188.

14. Agnihotri SA, Aminabhavi TM. Chitosan nanoparticles for prolonged delivery of timolol maleate. Drug Dev Ind Pharm. 2007;33(11):1254-1262.

15. Goyal R, Macri LK, Kaplan HM, Kohn J. Nanoparticles and nanofibers for topical drug delivery. J Control Release. 2016;240:77-92.

16. Cho IS, Park CG, Huh BK, et al. Thermosensitive hexanoyl glycol chitosan-based ocular delivery system for glaucoma therapy. Acta Biomater. 2016;39:124-132.

17. de la Fuente M, Ravina M, Paolicelli P, Sanchez A, Seijo B, Alonso MJ. Chitosan-based nanostructures: a delivery platform for ocular therapeutics. Adv Drug Deliv Rev. 2010;62(1):100-117.

18. Joshi GV, Patel HA, Kevadiya BD, Bajaj HC. Montmorillonite intercalated with vitamin B1 as drug carrier. Appl Clay Sci. 2009;45(4): 248-253.

19. Lin $\mathrm{FH}$, Chen $\mathrm{CH}$, Cheng WT, Kuo TF. Modified montmorillonite as vector for gene delivery. Biomaterials. 2006;27(17):3333-3338.

20. Oliveira AS, Alcantara ACS, Pergher SBC. Bionanocomposite systems based on montmorillonite and biopolymers for the controlled release of olanzapine. Mater Sci Eng C Mater Biol Appl. 2017;75:1250-1258.

21. Rajkumar S, Kevadiya BD, Bajaj HC. Montmorillonite/poly (L-lactide) microcomposite spheres as reservoirs of antidepressant drugs and their controlled release property. Asian J Pharm Sci. 2015;10(5):452-458.

22. Lin FH, Lee YH, Jian CH, Wong JM, Shieh MJ, Wang CY. A study of purified montmorillonite intercalated with 5-fluorouracil as drug carrier. Biomaterials. 2002;23:1981-1987.

23. Zheng JP, Luan L, Wang HY, Xi LF, Yao KD. Study on ibuprofen/ montmorillonite intercalation composites as drug release system. Appl Clay Sci. 2007;36(4):297-301.

24. Zheng J, Shan J, Fan Z, Yao K. Preparation and properties of gelatinchitosan/montmorillonite drug-loaded microspheres. J Wuhan Univ Technol Mater Sci Ed. 2011;26(4):628-633.

25. Hou D, Hu S, Huang Y, et al. Preparation and in vitro study of lipid nanoparticles encapsulating drug loaded montmorillonite for ocular delivery. Appl Clay Sci. 2016;119:277-283.

26. Huang Y, Tao Q, Hou DZ, et al. A novel ion exchange carrier based upon liposome-incorporated montmorillonite for ophthalmic delivery of betaxolol hydrochloride. Int J Nanomed. 2017;12:1-15.

27. Hou D, Xie C, Huang K, Zhu C. The production and characteristics of solid lipid nanoparticles (SLNs). Biomaterials. 2003;24(10):1781-1785.

28. Sharma K, Somavarapu S, Colombani A, Govind N, Taylor KM. Crosslinked chitosan nanoparticle formulations for delivery from pressurized metered dose inhalers. Eur J Pharm Biopharm. 2012;81(1):74-81.

29. Ameeduzzafar, Ali J, Bhatnagar A, Kumar N, Ali A. Chitosan nanoparticles amplify the ocular hypotensive effect of cateolol in rabbits. Int J Biol Macromol. 2014;65:479-491.

30. Hou DZ, Gui RY, Hu S, Huang Y, Pan YF, Song FL. Method for preparing novel drug delivery system for encapsulating montmorillonite loaded with chitosan. CN104491867A, 2014.

31. Giannola LI, de Caro V, Giandalia G, Siragusa MG, Cordone L. Ocular gelling microspheres: in vitro precorneal retention time and drug permeation through reconstituted corneal epithelium. $J$ Ocul Pharmacol Ther. 2008;24(2):186-196.

32. Chandasana H, Prasad YD, Chhonker YS, et al. Corneal targeted nanoparticles for sustained natamycin delivery and their PK/PD indices: an approach to reduce dose and dosing frequency. Int J Pharm. 2014; 477(1-2):317-325.

33. Wei G, Ding PT, Zheng JM, Lu WY. Pharmacokinetics of timolol in aqueous humor sampled by microdialysis after topical administration of thermosetting gels. Biomed Chromatogr. 2006;20(1):67-71. 
34. Gan L, Han S, Shen J, et al. Self-assembled liquid crystalline nanoparticles as a novel ophthalmic delivery system for dexamethasone: improving preocular retention and ocular bioavailability. Int J Pharm. 2010;396(1-2):179-187.

35. Liu R, Wang S, Fang S, et al. Liquid crystalline nanoparticles as an ophthalmic delivery system for tetrandrine: development, characterization, and in vitro and in vivo evaluation. Nanoscale Res Lett. 2016; 11(1):254

36. Talukder R, Fassihi R. Gastroretentive delivery systems: hollow beads. Drug Dev Ind Pharm. 2004;30(4):405-412.

37. Wadhwa S, Paliwal R, Paliwal SR, Vyas S. Chitosan and its role in ocular therapeutics. Mini Rev Med Chem. 2009;9(14):1639-1647.

38. Başaran E, Yazan Y. Ocular application of chitosan. Expert Opin Drug Deliv. 2012;9(6):701-712.

39. Silva MM, Calado R, Marto J, Bettencourt A, Almeida AJ, Gonçalves LMD. Chitosan nanoparticles as a mucoadhesive drug delivery system for ocular administration. Mar Drugs. 2017;15(12):370.
40. Hagino S, Itagaki H, Kato S, Kobayashi T, Tanaka M. Quantitative evaluation to predict the eye irritancy of chemicals: modification of chorioallantoic membrane test by using trypan blue. Toxicol In Vitro. 1991;5(4):301-304.

41. Lagarto A, Vega R, Guerra I, Gonzalez R. In vitro quantitative determination of ophthalmic irritancy by the chorioallantoic membrane test with trypan blue staining as alternative to eye irritation test. Toxicol In Vitro. 2006;20(5):699-702.

42. Nagarwal RC, Kumar R, Pandit JK. Chitosan coated sodium alginatechitosan nanoparticles loaded with 5-FU for ocular delivery: in vitro characterization and in vivo study in rabbit eye. Eur J Pharm Sci. 2012; 47(4):678-685.

43. Plager DA, Whitson JT, Netland PA, et al. Betaxolol hydrochloride ophthalmic suspension $0.25 \%$ and timolol gel-forming solution $0.25 \%$ and $0.5 \%$ in pediatric glaucoma: a randomized clinical trial. JAAPOS 2009; $13: 384-390$
International Journal of Nanomedicine

\section{Publish your work in this journal}

The International Journal of Nanomedicine is an international, peerreviewed journal focusing on the application of nanotechnology in diagnostics, therapeutics, and drug delivery systems throughout the biomedical field. This journal is indexed on PubMed Central, MedLine, CAS, SciSearch $®$, Current Contents $\AA /$ Clinical Medicine,

\section{Dovepress}

Journal Citation Reports/Science Edition, EMBase, Scopus and the Elsevier Bibliographic databases. The manuscript management system is completely online and includes a very quick and fair peer-review system, which is all easy to use. Visit http://www.dovepress.com/ testimonials.php to read real quotes from published authors.

Submit your manuscript here: http://www.dovepress.com/international-journal-of-nanomedicine-journal 